http://jmscr.igmpublication.org/home/ ISSN (e)-2347-176x ISSN (p) 2455-0450

crossref DOI: https://dx.doi.org/10.18535/jmscr/v8i3.68

Journal Of Medical Science And Clinical Research

\title{
A Rare Presentation of Skeletal Tuberculosis -Lumbar Spine and Metacarpals (Spina Ventosa)
}

\author{
Authors \\ Eleshwaram Naresh ${ }^{1}$, Gudepuvalasa Deepthi ${ }^{2}$, Kunche Alekhya ${ }^{3}$, L Lokanadha Rao ${ }^{4}$ \\ ${ }^{1,2,3}$ Junior Resident, Department of Orthopaedics, Andhra Medical College, Visakhapatnam, India \\ ${ }^{4}$ Professor, Department of Orthopaedics, Andhra Medical College, Visakhapatnam, India
}

\begin{abstract}
Introduction: Tuberculosis is an exceptionally infectious disease caused by Mycobacterium tuberculosis. TB primarily affects the lungs, but in some cases, it can spread to other parts of the body. When TB spreads, it's referred to as extrapulmonary tuberculosis (EPTB). One form of EPTB is bone and joint disease. Bone tuberculosis is simply a form of TB that affects the spine, the long bones, and the joints

Methods: A case report on an eight-year-old boy presented with multiple sinuses and ulcers over the right hand and spinal deformity since six months. The patient has a history of loss of appetite and loss of weight for one year, and restriction of movements in lumbar spine .patient was evaluated radiologically, and tuberculosis was confirmed by biopsy.

Results: The patient was put on anti-tubercular treatment initially and operated for correction of spinal deformity. ATT continued up to 9 months. Postoperatively, the patient regained lost movements, and the ulcers have healed completely.

Discussion: Spinal tuberculosis is a destructive form of the disease. It accounts for approximately half of all cases of musculoskeletal tuberculosis. Tuberculous dactylitis is one of the most frequent bacterial osteitis. It affects children more often than adults. Multiple bones are involved in children, and usually, only a single bone is involved in adults suffering from tuberculous dactylitis.

Conclusion: MRI and biopsy confirmed skeletal tuberculosis, the patient was put on ATT followed by CD fixation. Early diagnosis and prompt treatment will give an excellent outcome to patients.

Keywords: EPTB, Tuberculous Dactylitis, Biopsy, ATT, CD fixation.
\end{abstract}

\section{Introduction}

Bone and joint tuberculosis (TB) is an ancient disease. Evidence of osteoarticular TB has been detected in Egyptian mummies. ${ }^{(1)}$ Bone and joint TB comprises a group of severe infectious diseases whose incidence has increased in the past two decades, especially in underdeveloped countries, in part due to the AIDS epidemic. Tuberculous spinal infections should be suspected in patients with an insidious, progressive history of back pain and individuals from an endemic area, especially when the thoracic vertebrae are affected and a pattern of bone destruction with relative disc preservation and paravertebral and epidural soft tissue masses are observed. Atypical tuberculous osteoarticular manifestations involving the extraspinal skeleton, a prosthetic joint, or the trochanteric area, and nontuberculous 
mycobacterial infections should be considered in favorable epidemiological contexts. Surgery combined with prolonged specific antituberculosis chemotherapy is mainly indicated in patients with neurological manifestations or deformities and provides satisfactory results in most cases. ${ }^{(2)}$

\section{Case report}

An eight-year-old boy is complaining of multiple sinuses and ulcers over the dorsal and ventral aspect of the right hand and swelling for one-year Spinal deformity for six months.

\section{Clinical Presentation}

The patient had swelling of the right hand, which was insidious in onset and gradually increased in size, ulcers present over the dorsum of the right hand, which turned into discharging sinuses with foul-smelling pus associated with pain.

History of loss of appetite and loss of weight since one year. Gibbus deformity of spine present, which was insidious in the onset of six months duration. A similar sinus was present in the right axilla since one month.

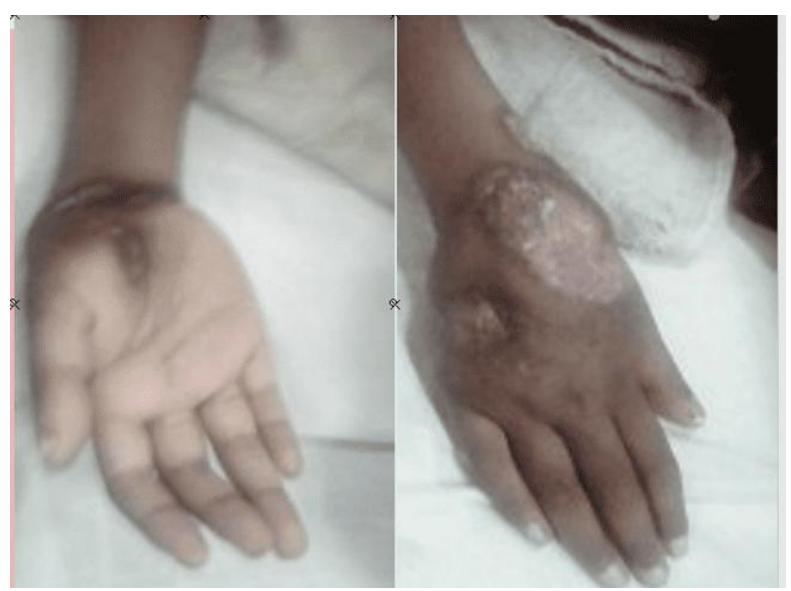

Figure 1: multiple sinuses and ulcers over the right hand

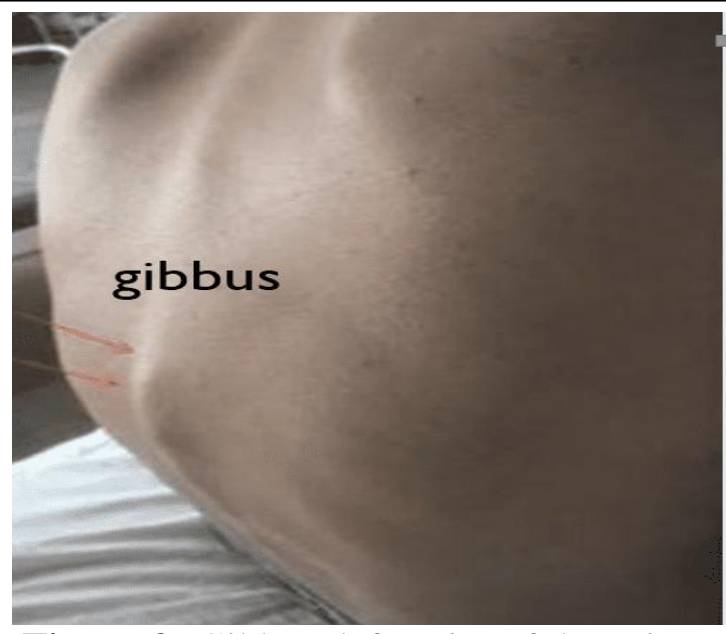

Figure 2: Gibbus deformity of the spine

\section{Investigations}

Routine Surgical profile and Biochemical tests were done with raised ESR. Mantoux test was positive (in duration $>10 \mathrm{~mm}$ ).

$\mathrm{X}$-ray right hand AP \& Oblique views show Diaphyseal expansile lesion of metacarpals, Thinning and Sclerosis of cortices.

Plain radiograph of the Dorso-Lumbar spine showed lysis of $\mathrm{L}_{2}-\mathrm{L}_{3}$ vertebrae and scoliotic deformity.

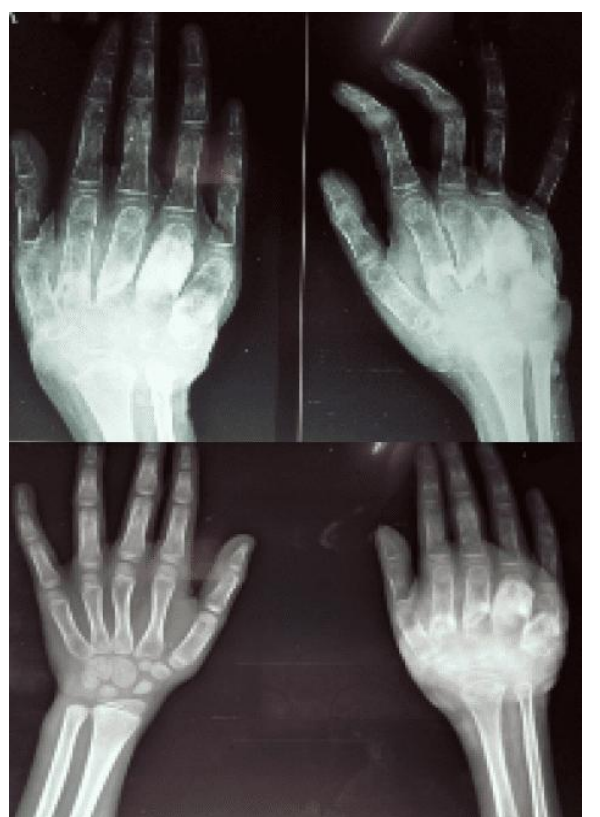

Figure 3: Expansile lesion with thinning \& Sclerosis of cortices. 


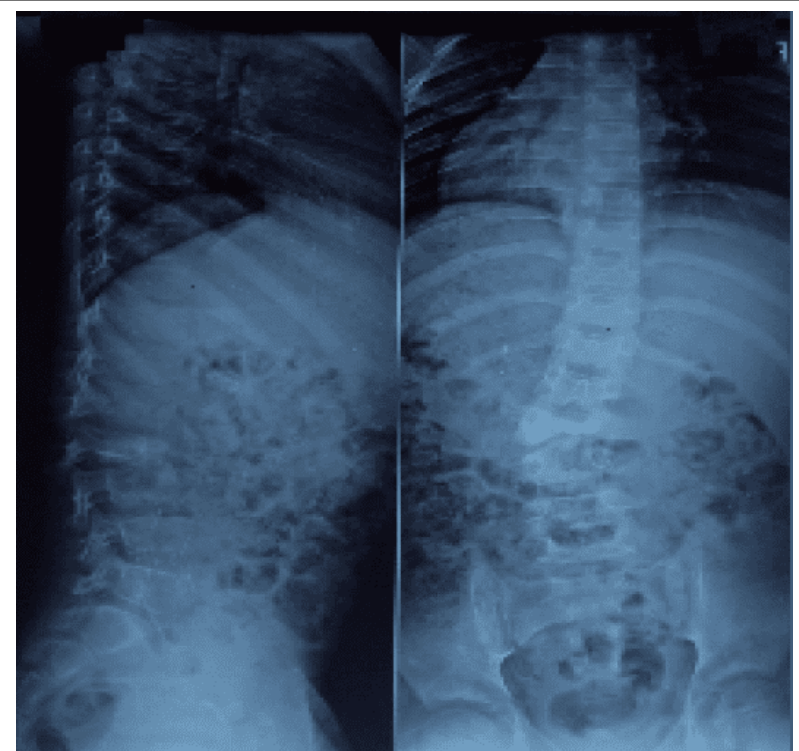

Figure 4: lysis of $\mathrm{L}_{2}-\mathrm{L}_{3}$ vertebrae with scoliotic deformity

MRI shows -Destruction of adjoining endplates of L2-L3bodies, pedicles on right side and intervertebral disc in between is seen with altered marrow signal associated with scoliotic convexity to left side Bilateral psoas abscess are seen on right side it is $55 * 46 \mathrm{~mm}$ in size and extending from L1 level toproximal thigh Both abscess are communicating with prevertebral abscess at L3 level.

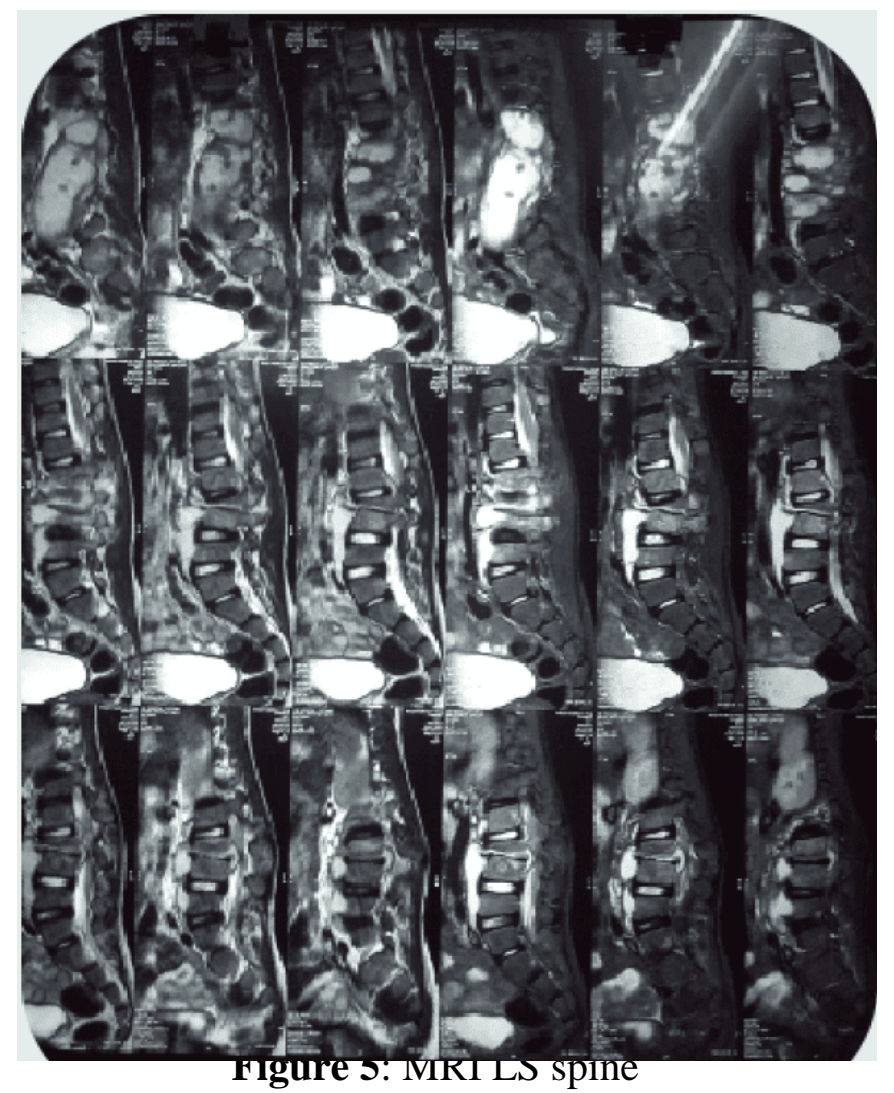

Tissue biopsy at lesions of the right hand showed skin with ulceration and subepidermal granuloma, the formation of foreign body giant cells, and the collection of chronic inflammatory cells.

Bone biopsy showed bony trabeculae with necrotic bony spicules \& granulomas composed of Langerhans giant cells, epithelioid cells, and caseous necrosisfoci of granular tissue and hemorrhagic areas.

A final diagnosis of Tuberculous spine with paraspinal cold abscess and scoliosis Involving L2-L3 Vertebrae with Tuberculous Dactylitis (Spina Ventosa) was made with the help of lab investigations, radiological and pathological findings.

\section{Treatment}

Anti-tubercular treatment: After the confirmation of the diagnosis, we have started ATT for the patient as per the guidelines of the RNTCP drug regime ${ }^{(3)}$ for this patient.

1. Isoniazid $100 \mathrm{mg}$

2. Rifampicin $200 \mathrm{mg}$

3. Pyrazinamide $500 \mathrm{mg}$

4. Ethambutol 300mg

Operative Management: Spine is stabilized by CD fixation done at the level of L1-L3under general anesthesia. 4.5 mmpoly-axial screws of $30 \mathrm{~mm}$ size were used. After the CD fixation, we continued ATT for up to 9 months.

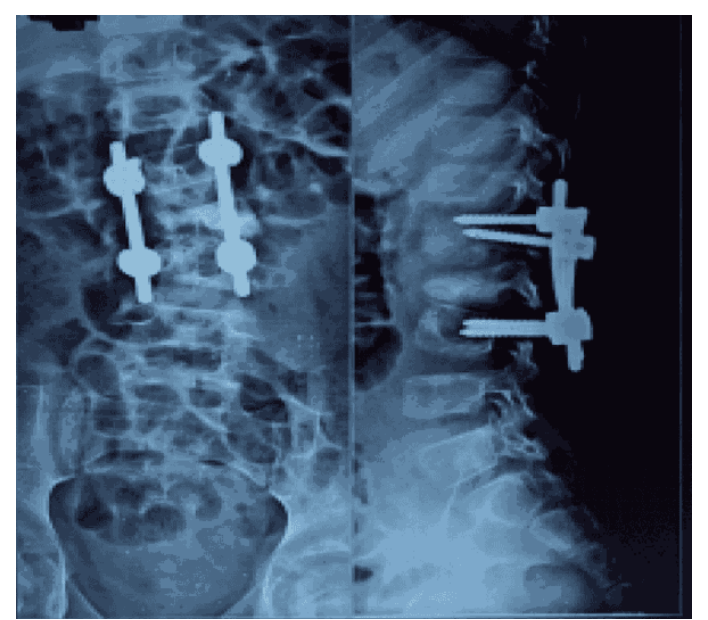

Figure 6: Post-op x-ray- spine after CD fixation 


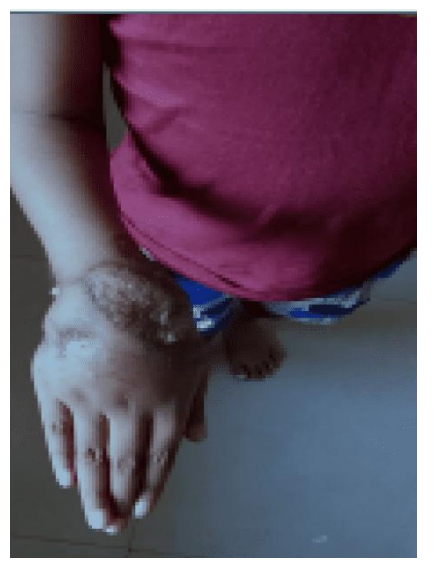

Figure 7: Healed sinuses and ulcers

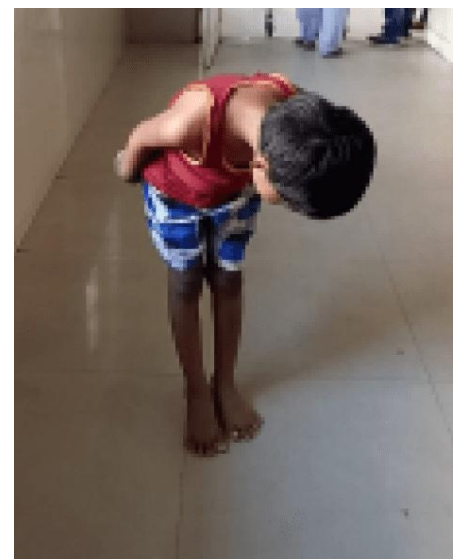

Figure 8: Improved movement of the spine

\section{Results}

The patient was operated for correction of spinal deformity and was put on anti-tubercular treatment for nine months postoperatively patient regained lost movements, and the ulcers have healed completely.

\section{Discussion}

Pott's disease is a form of tuberculosis that occurs outside the lungs, whereby disease is seen in the vertebrae. Tuberculosis can affect several tissues outside the lungs, including the spine, a kind of tuberculous arthritis of the intervertebral joints. The disease is named after Percivall Pott (1714-1788), a British surgeon. The lower thoracic and upper lumbar vertebrae are the areas of the spine most often affected. Pott's spine results from the haematogenous spread of tuberculosis from other sites, commonly the lungs. The infection then spreads from two adjacent vertebrae into the adjoining intervertebral disc space. If only one vertebra is affected, the disc is healthy, but if two are involved, the avascular disc cannot receive nutrients and collapse. In a process called caseous necrosis, the disc tissue dies, leading to vertebral narrowing and eventually to vertebral collapse and spinal damage. A dry soft tissue mass often forms, and superinfection is rare. ${ }^{(4)}$

Spina Ventosa is the term given for tuberculous dactylitis. Nearly $85 \%$ of the patients of Spina Ventosa are below six years of age. The bones of hands are more commonly involved than those of the feet. Proximal phalanx of the index and middle fingers are the most prevalent sites of involvement. Up to nearly $7 \%$ of children with pulmonary tuberculosis may develop this condition. Spread to the skeletal system is believed to occur via blood and lymphatics. ${ }^{(5)}$

\section{Conclusion}

Skeletal tuberculosis is relatively rare, but in the last few decades, the prevalence of this disease has increased in developing nations. While rare, skeletal tuberculosis is difficult to diagnose and can lead to severe problems if left untreated. Early diagnosis and prompt treatment will give an excellent outcome to patients.

\section{References}

1. Skeletal tuberculosis- UpToDate [Internet]. [cited 2020 Mar 8]. Available from: https://www.uptodate.com/contents/skeletaltuberculosis

2. Pigrau-Serrallach C, Rodríguez-Pardo D. Bone and joint tuberculosis. Eur Spine J. 2013 Jun;22(SUPPL.4):556.

3. SM Tuli. of the Skeletal System. 2010.

4. Pott disease - Wikipedia [Internet]. [cited 2020 Mar 8]. Available from: https://en.wikipedia.org/wiki/Pott_disease

5. Tuberculous dactylitis - Wikipedia [Internet]. [cited 2020 Mar 8]. Available from: https://en.wikipedia.org/wiki/Tuberculous_da ctylitis. 